\title{
Design and performance of future networks
}

\author{
Prosper Chemouil • Michael Menth • Deep Medhi • \\ Fabrice Guillemin
}

(C) Institut Télécom and Springer-Verlag 2010

In the past 10 years, the Internet has become the network capable of integrating all types of services (data, voice, video and TV). Nowadays, the IP layer appears as the convergence layer for ensuring connectivity between heterogeneous networks. Even though the shortcomings of the Internet in terms of quality of service, security and mobility are well understood in the technical literature, and in spite of initiatives flourishing all over the world (GENI in the USA, AKARI in Japan and various Research Programs in Europe), the predominance of the current Internet will certainly prevail in the near future.

Nevertheless, the emergence of new technologies, especially in optics and wireless, is substantially modifying the current landscape. The availability of smart phones and mobile computers in the mass market is going to accelerate the convergence of fixed and mobile networks. The architecture of the latter will be heavily modified by the introduction of IP as the convergence layer. In addition, the

\footnotetext{
P. Chemouil $(\bowtie)$

Orange Labs,

Issy-les-Moulineaux, France

e-mail: prosper.chemouil@orange-ftgroup.com

M. Menth

University of Tübingen,

Tübingen, Germany

e-mail: menth@informatik.uni-tuebingen.de

D. Medhi

University of Missouri-Kansas City,

Kansas City, MO, USA

e-mail: dmedhi@umkc.edu

F. Guillemin

Orange Labs,

Lannion, France

e-mail: fabrice.guillemin@orange-ftgroup.com
}

ability of smart terminals to support voice, video, data services and the evolution of the wireless technology towards even higher bit rates, is about to open the door to the transfer of massive volumes of traffic through the air interface. This will imply a deep rethinking of traffic engineering tools for both radio and mobile backhaul networks.

The situation is similar for the fixed access with the deployment of optical fibre. Very high bit rates $(1 \mathrm{Gbit} / \mathrm{s}$ or more for the downlink and at least $10 \mathrm{Mbit} / \mathrm{s}$ for the uplink) enable the transfer of huge amounts of traffic that have a great impact on backhaul and core networks. Moreover, the evolution of optical technology towards more flexibility with Dynamic Optical Circuit Switching and Optical Burst Switching should deeply modify the architecture of core networks where a better coordination between the IP and the physical layer will be necessary.

In parallel to technological evolutions, the Internet is the place of rapid emergence of new services and usage. As already observed with peer-to-peer networks, many overlay networks are rapidly emerging on top of the Internet, e.g. social networks that can potentially give rise to large amounts of traffic though the exchange of voluminous content (pictures, videos etc.) while requiring an acceptable quality of service level. The situation is similar for Over the Top players deploying services over the Internet and requesting quality of service and flexibility from the network. To facilitate this tremendous emergence of new usage and services, Content Distribution Systems will certainly be deployed on a large scale and will significantly modify the main traffic flows in networks, as we already witness such evolutions.

The above evolutions in terms of broadband applications, emergence of new technologies, and changes in usage, require the continual reappraisal of traffic manage- 
ment procedures and mechanisms as well as the development of new tools for understanding and modelling traffic and associated functions. These routines are crucial in the design of Future Networks and they were the central theme of the 21st International Teletraffic Congress that was held in Paris on September 15-17, 2009 (see ITC 21 website, http://www.i-teletraffic.org/itc21).

Following this successful conference, a special issue of the Annals of Telecommunications was initiated to address, in a broader context, design and performance issues of future networks. The open call for papers attracted 44 submissions among which 11 papers only were extended versions of papers presented at the ITC 21 conference. After a thorough peer review process, only nine papers of outstanding quality were selected. The diversity of the accepted papers shows the broad range of performance issues raised by future networks. They cover topics spanning application layer networks, IP networks, optical networks, access networks, wireless networks and consider routing, transport and resource management issues.

The first paper, "A New Statistical Approach to Estimate Global File Populations from Local Observations in the eDonkey P2P File Sharing System" by Patrick Brown and Sanja Petrovic is proposing a new statistical approach for the estimation of global population statistics from local observations. The method is inspired by "capture-recapture methods" used in biology and is computationally efficient and accurate. The authors implemented it in the peer-topeer ( $\mathrm{P} 2 \mathrm{P})$ context to estimate the number of replicas in the system, i.e. the size of the population of users possessing certain files, from local and time-limited observations. The method is applied on a measurement data set collected over several days on a residential network and results are compared with those from direct counting procedures. The knowledge of the population size in P2P systems is useful to set optimised system parameters, to derive other system statistics, or to predict system performance. As P2P systems may be very large, encompassing several millions of users, and highly distributed, estimating population sizes is a challenging task.

Next, the paper, "On the Interplay of Network Structure and Routing Strategies in Scale-Free Networks" by Walid K. Ghamry and Khaled M. F. Elsayed considers scale-free networks. In particular, they investigate the structural characteristics of network topologies and their effects on the network performance. The goal of the work is to understand the interplay between structural characteristics of network topologies and routing strategies.

The third paper, "On the Performance and Improvement of Alias Resolution Methods for Internet Core Networks" by Santiago Garcia-Jimenez, Eduardo Magana, Daniel Morato and Mikel Izal evaluates three core networks with results brought by several common IP alias resolution techniques. IP alias resolution is a critical part of IP network mapping, because it allows deriving router-level network topologies from network topologies measured with the traceroute tool and is unfortunately composed of IP addresses only. The authors focus on two important steps executed by IP alias resolution methods: reduction of the set of candidate IP address pairs to be tested as alias, and alias resolution. Due to the validation on real topologies of the three probed networks, this paper reveals the efficiency of the various IP alias techniques and suggests best practices.

Then the paper, "Compact Inter-domain Routing under Real-World Constraints" by Rolf Winter addresses the problem of Internet routing scalability. The natural growth of the Internet, operational practices, and a number of limitations in the BGP routing protocol create a large amount of state information in routers which requires a high signalling rate to keep it up to date and consistent. The author tackles this problem by proposing "Two Step Interdomain Routing" as an alternative Internet routing architecture that is inspired by the theoretical idea of compact routing. The author applied the new approach to today's Internet structure, constructing an Internet-scale AS-level topology that obeys real-world requirements such as business relationships. Several important metrics are derived to assess the viability of the proposed mechanism. In particular, the trade-off between required state space and path stretch is analysed.

The fifth paper, "Towards End-Host Based Identification of Competing Protocols against TCP in a Bottleneck Link" by Abdul Serwadda and Idris A. Rai investigates identifiable presence of UDP flows that compete against a TCP flow on a bottleneck link. Specifically, they consider the coefficient of variation and the power spectral density of throughput traces for this work. They observe that the coefficient of variation of throughput of TCP flows sharply increases due to competing UDP flows; furthermore, the spectra has been observed to be noisy with intensity proportional to the rate of the competing UDP flows. The results reveal distinct signatures that differentiate the presence of competing UDP flows from TCP flows independent of round trip time variations.

The next paper, "Quality Assurance of Voice Over WLANs (VoWLANs) with Differentiated Services" by Badis Tebbani, Kamel Haddadou, and Guy Pujolle considers the deployment of voice service over WLANs that raises several performance issues. The authors are proposing a session-based method that enables high-priority users to benefit from acceptable quality of service, by downgrading low-priority calls through codec change or, if performance gets worse, by stopping low-priority calls.

The seventh paper, "All-Optical Multipoint-to-Point Routing in WDM Mesh Networks" by Fen Zhou, Miklos 
Molnar, and Bernard Cousin addresses the routing and wavelength assignment problem to support multipoint-topoint communications. Since the number of wavelengths supported in a fibre link is limited, both the total cost and the link stress are important factors to consider. Keeping these in minds, two algorithms, Reverse Shortest Path Tree routing and k-Bounded Edge Disjoint Path Routing, are proposed and analysed for efficiency.

Next, the paper, "Parametric Distributions of Connection Lengths for the Efficient Analysis of Fixed Access Network" by Catherine Gloaguen, Florian Voss and Volker Schmidt presents a mathematical framework based on stochastic geometry that takes into account the strong dependency between the telecommunications infrastructure and the road systems infrastructure, in particular for access networks. In the context of the optical fibre deployment, this approach proves to be simple and efficient for a costeffective network planning.

Finally, the paper, "Maximum Weight Independent Sets in an Infinite Plane with Uni- and Bidirectional Interference Models" by Jarno Nousiainen, Jorma Virtamo and Pasi
Lassila addresses the theoretical problem of determining the maximum weight of independent sets in a wireless multihop network. Such a network is composed of interfering links between nodes randomly placed according to a Poisson process in the infinite plane, an independent set being composed of non-interfering links. Under various weight scenarios, the authors first study the problem when the mean number of neighbours within the transmission range went from zero to infinity. Then, for more general spatial processes, a practical solution is developed on the basis of the so called Moving Window Algorithm that allows a progressive exploration of the network and yields exact results. This algorithm can be used for any type of network realisations.

We would like to thank all the authors for their support in providing high-quality papers and all the reviewers for their diligent work that allowed selecting and improving the most appropriate contributions. We hope that the readers of the Annals of Telecommunications benefit from the ideas presented in this special issue and enjoy reading the contributions that address several challenges for future networks. 